\title{
Modelagem de Equações Estruturais e Satisfação do Consumidor: uma Investigação Teórica e Prática
}

\author{
Salomão Alencar de Farias \\ Rubens da Costa Santos
}

\section{RESUMO}

Este artigo é resultado de uma pesquisa que discute a aplicação da técnica estatística multivariada denominada análise de equações estruturais ou análise de caminhos, na avaliação de um esquema teórico da satisfação do consumidor, no segmento da terceira idade. Inicialmente, os autores dissertam sobre satisfação e análise de equações estruturais, apresentando uma revisão teórica destes temas, para mostrarem em seguida o modelo de satisfação do consumidor que serviu de base para as avaliações realizadas neste estudo. O principal objetivo foi o de investigar os antecedentes da satisfação do consumidor no segmento da terceira idade, visando a identificar quais os que são mais relevantes na determinação desta resposta pós-compra. Encontrou-se que dos antecedentes propostos pela teoria e avaliados neste estudo, os que mais contribuíram para a resposta de satisfação foram, em ordem de importância: desempenho, desconfirmação, justiça e emoções.

Palavras-chaves: satisfação do consumidor; modelagem de equações estruturais; segmento da terceira idade.

\begin{abstract}
This paper is the result of a quantitative research project that discusses the application of the multivariate technique structural equation modeling or path analysis, to the assessment of a theoretical customer satisfaction framework, using a sample of elderly consumers in Brazil. The authors discuss customer satisfaction and structural equation modeling, and present a literature review of both topics. The customer satisfaction model that was used in the modeling process is then shown. The main objective was to investigate what leads to customer satisfaction among the elderly, trying to identify which factors are the most relevant to the post-purchase response in this specific market segment. It was found that of the antecedents proposed by customer satisfaction theory, the ones that most contributed to the satisfaction response in this segment were: performance, disconfirmation, justice and emotions.
\end{abstract}

Key words: customer satisfaction; structural equation modeling; elderly segment. 


\section{APREsentaÇÃo}

Nos encontros da ANPAD realizados em 1997 e 1998, a área de marketing apresentou nove artigos que lidam diretamente com o tema satisfação do consumidor (e.g., Farias et al., 1997; Rossi e Slongo, 1997; Farias e Santos, 1998; Prado e Farias, 1998; Urdan e Rodrigues, 1998). Em revistas nacionais, destacam-se os artigos de Prado e Marchetti (1997), publicado na RAUSP, e o de Grandis Rojo (1998), publicado na RAE, que lidam com a satisfação do consumidor em supermercados. Em publicações internacionais, diversos autores têmse interessado pelo tema satisfação do consumidor, a exemplo de Yi (1991), que efetivou uma extensiva revisão das teorias da satisfação, publicada na Review of Marketing da AMA, e Oliver (1997) que lançou um livro com ênfase comportamental na satisfação do consumidor. Mais recentemente, Fournier e Mick (1999) publicaram uma investigação fenomenológica e longitudinal sobre o tema no Journal of Marketing.

O presente artigo discute o uso da modelagem de equações estruturais (SEM) na avaliação de um esquema de satisfação do consumidor no segmento de consumidores com idade acima dos 55 anos, no que concerne ao consumo de serviços de hotelaria. A pesquisa justifica-se pela importância da temática satisfação para o marketing. Já o segmento escolhido para a operacionalização do estudo é um dos que mais crescem no Brasil, aparentando ter uma parcela de consumidores da terceira idade atrativa para as empresas que desejarem explorar este nicho, em termos de marketing. A modelagem de equações estruturais é ferramenta utilizada para uma melhor compreensão deste fenômeno.

\section{SatisfaÇão do Consumidor}

A satisfação do consumidor vem sendo encarada como fator crucial para o sucesso dos mais variados tipos de organizações, especialmente por influenciar diretamente a lealdade à marca, repetição de compras, comunicação boca-aboca positiva, lucratividade e participação de mercado (Oliver, 1997). São dimensões que, certamente, toda a organização que deseje continuar com sucesso no mercado gostaria de saber como influenciar e controlar. Czepiel, Rosenberg e Surprenant (1980) citam o ano de 1972 como a data em que o estudo da satisfação do consumidor surgiu como campo legítimo de pesquisa, embora Cardozo 
(1965) seja o autor citado como o pioneiro nesta área. A formação ou operacionalização da satisfação do consumidor ocorre em ambiente dinâmico, que exige constante atualização e enriquecimento da teoria e da prática que lidam com este construto tão relevante para o marketing e para o comportamento do consumidor (Ewald, 1993).

A incorporação de novas variáveis que se apresentam no ambiente dinâmico, citado no parágrafo anterior, junto a um ou mais esquemas de satisfação já existentes, pode levar a um melhor entendimento e/ou explicação deste fenômeno. Para se ter uma idéia, Oliver (1980) propunha que a satisfação do consumidor era função apenas das expectativas e da desconfirmação. Mais recentemente, além dessas duas variáveis, este mesmo autor afirma que performance, atribuição (local, estabilidade e controlabilidade), eqüidade (justiça e preferência) e emoções são determinantes possíveis da satisfação (Oliver, 1997).

O esquema teórico apresentado na metodologia estabelece relações prováveis entre os paradigmas existentes na literatura, que explicam a satisfação do consumidor. Não foram encontrados na literatura especializada, estudos que investigassem este modelo no segmento da terceira idade, para verificar se todos os antecedentes são realmente significativos neste nicho, considerando que o processo de envelhecimento causa mudanças no comportamento do consumidor (Moschis, 1992). Os próximos itens apresentam comentários sobre os antecedentes da satisfação do consumidor, de forma resumida.

\section{Paradigmas da Satisfação do Consumidor}

Tendo início com o trabalho preliminar de Cardozo (1965), como se mencionou, o campo de estudo da satisfação do consumidor amadureceu para uma respeitável corrente de pesquisa. De fato, mais do que outros em disciplinas relacionadas, os pesquisadores do comportamento do consumidor avançaram e testaram o processo de satisfação. Cinco teorias do processo de satisfação foram propostas: desconfirmação da expectativa, teoria da assimilação, teoria da eqüidade, teoria da atribuição e teoria do desempenho.

Cardozo (1965, p. 244) cita que "a satisfação do consumidor com um produto leva à repetição de compra, aceitação de outros produtos na mesma linha de produto, bem como ao boca-a-boca favorável. Se isto estiver correto, então o conhecimento dos fatores que afetam a satisfação do consumidor é essencial".

Os antecedentes da satisfação existentes na literatura são sumariados em seguida. 


\section{Desconfirmação da Expectativa (Expectativa e Desconfirmação)}

Com raízes na psicologia social e no comportamento organizacional, a desconfirmação (não confirmação) da expectativa é na realidade dois processos que consistem na formação de expectativas e na sua desconfirmação mediante a comparação de desempenho. Como apresenta Oliver (1980), acredita-se que os consumidores formam expectativas a respeito do desempenho das características dos produtos antes da compra. A compra e o uso subseqüente revelam os níveis de desempenho reais, que são comparados aos níveis de expectativa pelo uso de parâmetros do tipo melhor do que ou pior do que esperado, com base na experiência própria dos consumidores. O julgamento que resulta desta comparação é rotulado de desconfirmação negativa se o produto é pior do que o esperado; desconfirmação positiva, se melhor do que esperado; simples confirmação ou desconfirmação zero, se ocorre o esperado.

O nível da expectativa parece providenciar a base, ao redor da qual os julgamentos relacionados à desconfirmação são feitos. Quanto mais alta ou baixa a expectativa do consumidor, mais alto ou baixo o julgamento das satisfações subseqüentes, ceteris paribus. $\mathrm{O}$ prazer de uma desconfirmação positiva acentua um julgamento de satisfação, enquanto o desapontamento de uma desconfirmação negativa o diminui (Oliver, 1980).

Comparando-se a expectativa e a desconfirmação, acredita-se que esta última seja a variável mais forte na satisfação, talvez porque o efeito da expectativa tenha tempo para enfraquecer através do intervalo da compra; no entanto pode ser instrutivo considerar que alguns consumidores são mais influenciados pelas expectativas, que outros o são pela desconfirmação, e ainda que outros usam ambos os construtos como efeitos separados na resposta de satisfação.

\section{Teoria da Assimilação}

A teoria da assimilação, segundo Oliver e DeSarbo (1988), oferece a base para uma tendência à resposta orientada nas expectativas. Dividindo alguns elementos com a teoria da dissonância cognitiva, esse quadro teórico assume que os indivíduos relutam em aceitar discrepâncias com relação a posições previamente assumidas e, deste modo, assimilarem julgamentos com respeito aos seus sentimentos iniciais para um objeto ou evento. Assim, para as pessoas influenciadas pelas expectativas, pode-se esperar que respondam com níveis de satisfação similar em harmonia com suas expectativas.

Este paradigma está baseado na habilidade individual de o consumidor expli- 
car ou racionalizar as aparentes discrepâncias percebidas na experiência de consumo e no pós-compra (Oliver, 1997).

\section{Teoria da Eqüidade}

De modo geral, a teoria da eqüidade sugere que as partes envolvidas em uma troca se sentirão tratadas com justiça e assim satisfeitas, se a proporção da saída com relação à entrada é de alguma forma justa. A satisfação existe quando a pessoa central (o consumidor) percebe que a sua razão entrada/saída é proporcional à do seu parceiro na troca (Oliver e DeSarbo, 1988). Desta forma, quando as entradas não são proporcionalmente altas para a pessoa central, a satisfação deve aumentar com o incremento das saídas, relativamente à do outro indivíduo e tende a decair, quando as saídas decrescem, de maneira comparativa.

Para Oliver e Swan (1989) o conceito da eqüidade é entendido como fator adicional na resposta pós-compra, a qual tem sido dominada pelo paradigma da desconfirmação das expectativas. Esses autores sugerem que os processos de eqüidade e de desconfirmação são complementares nas respostas dos consumidores.

\section{Teoria da Atribuição}

Esta teoria propõe que as saídas podem ser entendidas como sucesso ou falha, trazendo à tona inferências de causalidade por meio de três dimensões: local da causa (fonte interna contra externa da causa); estabilidade (variabilidade) da causa da saída; e a controlabilidade. Dessas dimensões, o local da causa (interna/externa) parece o fator principal na satisfação do consumidor. Por exemplo, o consumidor pode colocar a culpa da sua insatisfação no vendedor que sugeriu uma opção de produto (Oliver e DeSarbo, 1988).

Segundo Oliver (1997) há uma clara tendência de o consumidor atribuir a causa da avaliação negativa de uma experiência de compra a uma fonte externa. De outro lado, quando a experiência é positiva, o consumidor, em uma freqüência três vezes maior, tende a atribuir o sucesso a si próprio.

\section{Desempenho}

O efeito do desempenho do produto/serviço na satisfação é considerado para determinar se este é capaz de subjugar as tendências das respostas psicológicas discutidas. Embora a relação desempenho-satisfação pareça aceita como verda- 
deira, o efeito do desempenho é ambíguo para produtos que não possuem dimensões objetivas ou instrumentais de desempenho (dimensões intangíveis): a performance do produto tende a ser um dos antecedentes da satisfação (Oliver e DeSarbo, 1988).

Com relação à qualidade percebida (performance), autores como Parasuraman, Zeithaml e Berry (1985) vêm desenvolvendo, desde a década de 80, estudos que buscam uma melhor forma de se mensurar tal dimensão no consumo de serviços.

\section{Emoções no Consumo}

As emoções no consumo se referem ao conjunto de respostas hedônicas, que surgem especificamente durante o uso de um produto ou experiência de consumo, sendo descritas tanto por "categorias distintivas da experiência emocional e expressão (por exemplo, raiva e medo) ou por dimensões estruturais que ressaltam categorias emocionais, tais como prazer/desprazer, relaxamento/ação ou calma/excitação" (Westbrook e Oliver, 1991, p. 85).

Oliver (1989) propôs cinco diferentes estados emocionais qualitativos para o caso de satisfação. Ordenados pelo aumento do favorecimento e contribuição para a satisfação, eles são: aceitação, alegria, alívio, interesse/excitação e prazer. Para a insatisfação, os grupos emocionais, em ordem decrescente de favorabilidade, são: tolerância, tristeza, arrependimento, agitação e ultraje. Esse autor enfatiza que evidências empíricas destas propostas ainda não surgiram.

Alguns modelos específicos dos sentimentos, os quais incluem emoções, são geralmente descritos como circumplex, porque as várias emoções expressadas pelos indivíduos podem ser definidas em duas dimensões (intensidade é normalmente a terceira) na periferia de um círculo (Watson e Tellegen, 1989; Plutchik apud Oliver, 1989).

Com base na análise de quatro diferentes tipologias de emoção, e mais tarde de quatro diferentes métodos de camadas, Russell (apud Oliver, 1989) propôs que duas dimensões primárias abordam todos as emoções, denominadas prazerosas/desprazerosas e excitação/chateação. Tipicamente as dimensões expressas no estudo das emoções no consumo são estabelecidas pela aplicação da análise fatorial a um conjunto de variáveis emocionais (Westbrook e Oliver, 1991). Izard (1977) definiu um grupo de dez emoções, sem considerar dimensões específicas para o estudo desta faceta comportamental do consumidor.

A satisfação do consumidor pode ser explicada, considerando os paradigmas 
apresentados anteriormente. Uma das formas de se estudar a satisfação na prática, é por meio da sua mensuração e conseqüente identificação de variáveis que venham a predizê-la. Desse modo, pode-se definir ações específicas e efetivas, com o objetivo de manutenção ou incrementação da satisfação. De fato, a satisfação tem sido estabelecida como um agente de causa chave, responsável pela mudança de atitude baseada na experiência.

Nos próximos itens os autores apresentam uma revisão teórica sobre a modelagem de equações estruturais, com o objetivo principal de familiarizar o leitor com a técnica estatística multivariada, utilizada nas análises desta pesquisa, contribuindo para uma melhor compreensão dos aspectos metodológicos que são discutidos logo em seguida.

\section{Modelagem de Equaçóes Estruturais}

A modelagem de equações estruturais (structural equations modeling - SEM) pode ser vista, segundo Klem (1995), como extensão da regressão múltipla, se for considerado que na aplicação da regressão o pesquisador está interessado em prever uma única variável dependente, enquanto na SEM há mais de uma variável dependente. A preocupação nesta técnica é com a ordem das variáveis. $\mathrm{Na}$ regressão X influencia Y; na SEM X influencia Y e Y influencia Z. Uma das características básicas da SEM é que se pode testar uma teoria de ordem causal entre um conjunto de variáveis. No caso da satisfação do consumidor, por exemplo, a teoria propõe que a performance influencia a desconfirmação e que a desconfirmação pode levar à satisfação. É possível verificar tal relação causal com o uso da SEM. Esta técnica oferece ao pesquisador a possibilidade de investigar quão bem as variáveis preditoras (predictors) explicam a variável dependente (criterion) e, também, qual das variáveis preditoras é a mais importante. Isto também pode ocorrer com o uso da regressão, embora deve ser lembrado que aqui se pode ter mais de uma variável dependente em um único modelo (Maruyama, 1998).

Klem (1995) e Maruyama (1998) afirmam que dois resultados principais podem ser esperados de uma análise com a aplicação da SEM. Primeiro, uma estimativa da magnitude dos efeitos estabelecida entre variáveis é oferecida. Estas estimativas estão condicionadas ao fato de o modelo especificado (diagrama) estar correto. Segundo, é possível testar se o modelo é consistente com os dados observados. Se o modelo e os dados são consistentes, pode-se dizer que este é plausível, embora não se possa afirmar que este é correto (Klem, 1995). 
Para Maruyama (1998) a análise de caminhos (path analysis) está relacionada a modelos com fluxo causal direcional único, em que as medidas de cada variável conceitual são perfeitamente confiáveis. Partindo desta premissa, acreditase que não existe erro de medida (mensuração) ou de especificação (operacionalização) das variáveis. Isto é, cada medida é vista como exata manifestação da variável teórica. Certamente, nas ciências sociais, assumir que há perfeita confiabilidade é irreal. Este fato restringiu, por muito tempo, a aplicação desta técnica de análise de dados neste campo da ciência. Uma das soluções parciais encontradas para este problema foi a inclusão de variáveis não observadas, ou latentes e/ou erros no modelo teórico. Estes indicadores revelam a quantidade de variância não explicada pelas variáveis exógenas (independentes) especificadas.

Segundo Maruyama (1998), os métodos de SEM devem ter início em um modelo conceitual que especifique as relações entre um conjunto de variáveis. A teoria oferece o ponto central desta técnica. Como foi citado, a SEM oferece estimativas da força de todas as relações hipotetizadas em um esquema teórico. As informações disponibilizadas referem-se tanto ao impacto de uma variável sobre a outra como da relação de uma influência indireta, de uma variável posicionada entre duas outras, denominada interveniente ou mediadora.

Hair Jr. et al. $(1995,1998)$ afirmam que a teoria oferece a racionalização para quase todos os aspectos da SEM. Para estes autores, a SEM é mais um método de análises confirmatórias, guiado mais pela teoria do que por resultados empíricos.

\section{Histórico da SEM}

A SEM tem sido rotulada de modelagem de caminhos (path modeling), análise de caminhos (path analysis), e análise de variáveis latentes de equações estruturais (latent variable analysis of structural equations). Além disso, esta técnica tem sido referida até pela denominação de alguns programas como, por exemplo, análise LISREL (Jöreskog e Sörbom, 1993). Para Maruyama (1998) o termo particular utilizado reflete tanto a filosofia do pesquisador quanto o período em que este aprendeu a utilizar esta análise. Historicamente esta técnica foi denominada path analysis devido ao trabalho de Wright (apud Maruyama, 1998). Na década de 70, com o avanço dos computadores e outros aplicativos que tornaram possível a realização de análises complexas, o termo modelagem causal foi utilizado. Maruyama (1998) afirma que o termo modelagem causal foi rejeitado por alguns cientistas sociais que não aceitavam o uso da denominação causal em estudos não experimentais. Assim, a denominação equações estruturais passou a ser usada. As relações nesta abordagem são definidas por uma 
série de equações que descrevem as estruturas hipotetizadas das relações. A especificação análise de equações estruturais ou modelagem de equações estruturais é relacionada a isto. A força e a convicção com as quais um pesquisador pode assumir a causalidade entre duas variáveis baseiam-se na justificativa teórica que apóia as análises (Hair Jr. et al., 1998).

Para Klem (1995) a análise de caminhos foi usada pela primeira vez em 1918 por Sewall Wright, um geneticista que resolveu equações simultâneas para desvendar influências genéticas através de gerações. Ele desenvolveu um modelo unidirecional, em que as causas e as saídas eram conhecidas, e a causalidade era estipulada em uma única direção, sem feedback. Este modelo é conhecido como unidirecional ou recursivo. É o único que pode ser apropriadamente chamado de análise de caminhos (Maruyama, 1998). Nas ciências sociais, apenas em 1960, sociólogos, psicólogos, economistas e cientistas políticos começaram a escrever sobre esta técnica, trazendo-a para a atenção dos cientistas sociais. Blalock (1964) e Duncan (1966), citados por Klem (1995) e Maruyama (1998), são considerados os pioneiros na aplicação desta técnica na área das ciências sociais. Os programas mais conhecidos que desempenham a SEM atualmente são: o LISREL (Jöreskog e Sörbom, 1993), EQS (Bentler, 1989), e o Amos (Arbuckle, 1997; SPSS, 1998). Todos têm em comum o fato de utilizarem modelos de regressão linear nas análises efetuadas para verificar as relações hipotetizadas entre variáveis. Além destes programas, Hair Jr. et al. (1998) citam o PROC CALIS of SAS, o COSAN e o LVPLS. Loehlin (1998) acrescenta o MX, o RAMONA e o SEPATH à lista de programas que realizam a SEM.

\section{O Processo de Modelagem}

O ponto inicial para a aplicação da SEM é a teoria utilizada pelo pesquisador a respeito das relações causais entre um conjunto de variáveis. O pesquisador deve estar bem fundamentado na teoria na especificação das relações de causa. Assumindo isto como premissa, expressa-se esta teoria de modo formal e claro. O modelo é apresentado tanto em palavras quanto em diagrama. O diagrama pode ser entendido como um grupo de afirmativas que resumem um conjunto de hipóteses. Segundo Hair Jr. et al. (1998), o erro mais crítico no desenvolvimento de modelos com base teórica é a omissão de uma ou mais variáveis independentes, um problema conhecido como erro de especificação. Para estes autores todos os modelos de equação estrutural são acometidos deste erro de alguma forma, considerando-se que um construto ou indicador em potencial pode ser excluído.

Para efetivar-se a análise, faz-se necessária a existência de dados para cada variável do modelo. As variáveis devem ser mensuradas em escala intervalar ou, possivelmente, em escala ordinal, que possa ser tratada como intervalar. O número 
de casos necessários depende da complexidade do modelo. Sugerem-se 200 a 300 casos para cada modelo (Klem, 1995; Maruyama, 1998). Para Hair Jr. et al. (1998) deve-se ter de 5 a 10 respondentes por parâmetro no modelo. O número mínimo de elementos que compõem a amostra deve ficar entre 100 e 150 , quando se utiliza a estimação de máxima verossemelhança (maximum likehood) no cálculo dos parâmetros.

Existem dois tipos de variáveis em um modelo de SEM (Loehlin, 1998): variáveis endógenas e variáveis exógenas. Os valores das variáveis endógenas são explicados por uma ou mais variáveis exógenas do modelo. Os valores das variáveis exógenas são assumidos como dados, isto é, o modelo não tenta explicálos. Esta distinção é similar à feita entre variáveis dependentes (endógenas) e independentes (exógenas) da análise de regressão. Entretanto, na SEM, uma variável pode ser tanto dependente quanto independente. A regra é esta: se uma variável é dependente em alguma parte do modelo, então ela é endógena (Klem, 1995). Hair Jr. et al. (1998) argumentam que o diagrama deve ser definido em termos de construtos, para então buscar-se variáveis que irão mensurá-los. A regra geral é que cada construto é definido por um número de indicadores entre 5 e 7, embora quando se lida com escalas preexistentes, isto não seja válido. Os construtos podem ser exógenos ou endógenos. Cada construto endógeno constitui uma variável dependente nas equações estruturais.

\section{Convenções no Desenho de Diagramas}

O programa estatístico considerado para discussão neste estudo é o Amos 3.6 (Arbuckle, 1997). Com o Amos Graphics é possível realizar as análises a partir do diagrama, sem a necessidade de indicar as equações. É um programa compatível com a plataforma Windows e, atualmente, é o pacote utilizado pelo SPSS (1998) para este tipo de análise. Anteriormente o LISREL era o programa comercializado por esta empresa. A notação utilizada no Amos é praticamente a mesma comentada por Klem (1995) e Maruyama (1998).

Segundo Arblucke (1997), o desenho das variáveis no diagrama a ser reconhecido pelo Amos segue o especificado: os retângulos representam as variáveis observadas (mensuradas); as elipses representam as variáveis não observadas (latentes) ou os erros; uma seta reta com uma única ponta indica o caminho ou a relação de causa entre duas variáveis; e uma seta curva com duas pontas entre duas variáveis representa uma covariância. Para Maruyama (1998) as setas curvas com duas pontas representam as relações entre as variáveis exógenas, que são tidas como existentes, mas não são explicadas no modelo. Os diagramas devem ser desenhados de modo que o fluxo causal seja da esquerda para a direita. 
Os modelos representados nos diagramas utilizados na SEM podem ser classificados, primariamente, em dois tipos, dependendo do direcionamento das setas. Para os modelos em que as causalidades são propostas em uma única direção, a denominação utilizada é recursivo (recursive), podendo o modelo ser classificado em totalmente recursivo ou não recursivo. Quando é totalmente recursivo, cada variável tem efeito direto nas outras. Em modelo que não é totalmente recursivo, uma ou mais das ligações diretas permitidas pela ordem causal não são especificadas no diagrama. Quando o fluxo causal tem mais de uma direção, os modelos são denominados não recursivos (Loehlin, 1998).

\section{Cálculo dos Coeficientes}

Segundo Klem (1995) a forma mais simples e tradicional de calcular os coeficientes das relações entre variáveis exógenas e endógenas é mediante o uso de regressão múltipla. Uma análise de regressão é necessária para cada variável endógena no modelo. O modelo procura explicar a variância nestes indicadores. De acordo com Maruyama (1998), os efeitos diretos das variáveis exógenas nas endógenas são estimados por meio da análise de regressão OLS (ordinary least squares): cada variável endógena deve ser vista como tendo sua própria equação de regressão que descreve a estrutura das relações entre variáveis, isto é, equações estruturais (cada equação de regressão é uma equação estrutural).

O efeito direto entre duas variáveis é revelado no diagrama na finalização dos cálculos por meio do Amos (Arbuckle, 1997). Os efeitos indiretos - os caminhos que se estendem através de variáveis intermediárias - devem ser calculados da seguinte forma: localizam-se todas as rotas indiretas em que o efeito flui de uma variável para outra e, posteriormente, para cada rota, multiplicam-se os coeficientes. Finalmente, acrescentam-se os produtos encontrados para obter-se o efeito indireto. A variável que intervém entre duas, em um modelo de SEM, funciona como mediadora. O Amos apresenta, ainda, uma opção para o cálculo do efeito total (direto e indireto) entre variáveis.

Para cada par de variáveis no modelo há uma correlação implícita. Esta correlação, segundo Klem (1995), é a soma de quatro componentes: o efeito direto (se existente), a soma dos efeitos indiretos (se existentes) e de dois outros possíveis componentes, quais sejam, a soma dos efeitos espúrios e a soma dos efeitos não analisados. Uma correlação implícita no modelo não necessita ter todos esses componentes. Um efeito espúrio entre variáveis surge, quando elas têm uma causa comum. É caracterizado por um caminho que vai em sentido contrário à direção das setas. Já um efeito não analisado é aquele que envolve uma seta curva com duas pontas (correlação), entre variáveis exógenas. Este efeito é tido como não analisado, por não envolver uma relação causal, mas sim uma correla- 
ção, onde a ordem causal não é especificada. Para indicar o residual e o erro da variância, o Amos (Arbuckle, 1997) oferece a opção da inclusão de uma variável erro, que indica a variância não explicada no modelo. Lembra-se que, na regressão múltipla, cada caso tem um residual que é diferente entre o valor observado para a variável dependente e para a variável independente: o residual é o erro na predição.

Klem (1995) afirma que, ao analisarem-se os resultados de uma análise de caminhos, o pesquisador deve considerar a possibilidade da existência do seguinte: erros de medidas nas variáveis observadas; presença de erros de especificações no modelo; e presença de multicolinearidade. Finalmente, alerta para a necessidade de averiguar-se se o número de casos é suficiente. "Uma regra geral é que se tenha de 5 a 10 observações para cada parâmetro"(Klem, 1995). Dessa forma, cada seta no diagrama contém um parâmetro, incluindo as setas para os residuais.

\section{Metodologia da Pesquisa}

Enfatiza-se que este artigo é resultado de uma pesquisa que lida com a satisfação do consumidor e que utiliza a ferramenta estatística modelagem de equações estruturais em suas análises. Para sua operacionalização, estudou-se o indivíduo do segmento da terceira idade e o serviço de hotelaria. Os autores tiveram a intenção de demonstrar como a análise de equações estruturais (análise de caminhos), pode auxiliar na identificação de atributos relevantes na avaliação de um serviço, em termos de satisfação. O objetivo principal está centrado em avaliarem-se simultaneamente os determinantes da satisfação do consumidor no segmento da terceira idade, no atinente ao consumo de serviços de hotelaria, conforme já especificado.

A pergunta a que se buscou responder foi: avaliando-se simultaneamente os determinantes da satisfação do consumidor da terceira idade, quais os que mais contribuem para explicar este construto?

O modelo teórico proposto por Oliver (1997) e adaptado para esta pesquisa é apresentado a seguir, onde constam seis antecedentes da satisfação do consumidor: eqüidade, performance, expectativa, desconfirmação, emoções e atribuição, bem como as relações propostas entre estas variáveis. 
Figura 1: Determinantes da Satisfação do Consumidor

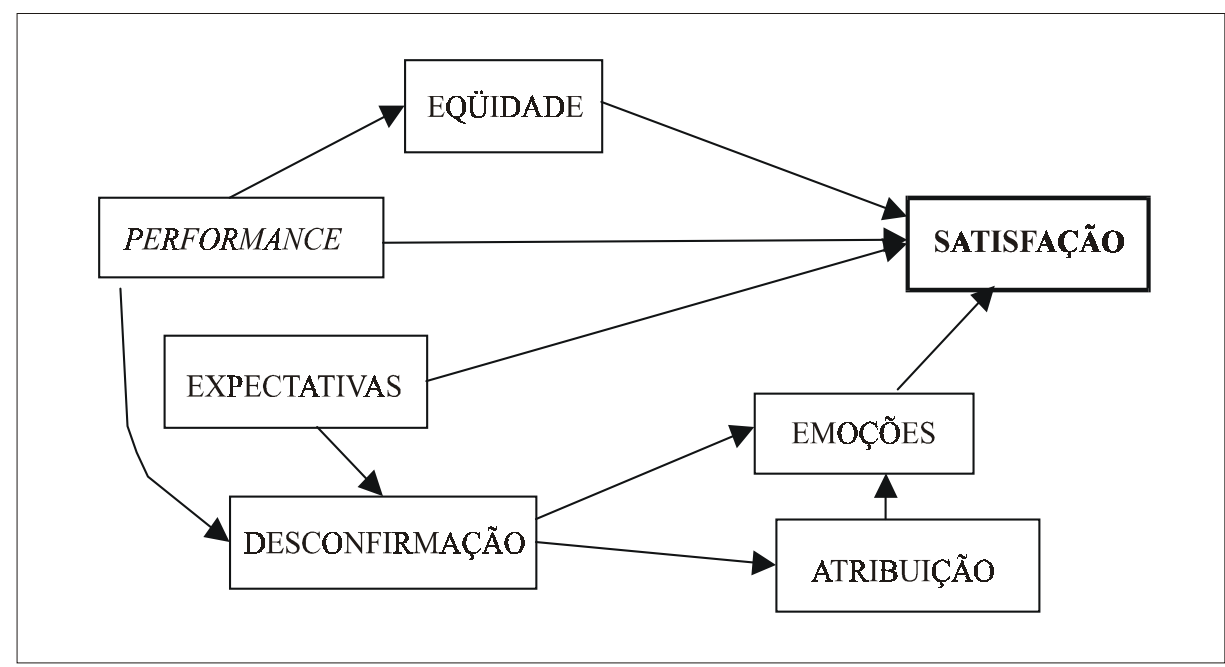

Fonte: adaptado de Oliver (1997, p. 337).

\section{Dimensões Mensuradas}

A identificação dos atributos de satisfação que se utilizou da técnica do incidente crítico (Flanagan, 1954; Bitner, Nyquist e Booms, 1985), aplicada por meio de entrevistas pessoais a uma amostra inicial de hóspedes, fez-se necessária, uma vez que, para a elaboração do questionário a teoria recomenda avaliarem-se atributos de satisfação, considerando o ponto de vista dos consumidores nos blocos referentes às expectativas, à desconfirmação e à performance, para fins de análises e comparações. Desse modo, na primeira versão do questionário, havia 3 blocos com itens que se propunham investigar atributos de satisfação do serviço oferecido pelos hotéis, além de 5 outros, que abordaram a satisfação, a eqüidade, a atribuição, as emoções e variáveis demográficas ${ }^{(1)}$.

Dispondo-se do questionário elaborado, o próximo passo foi o pré-teste deste instrumento, agora na fase quantitativa da pesquisa. Um grupo composto de 20 entrevistados foi utilizado neste processo. O bloco referente à atribuição apresentou problemas de compreensão junto a esta amostra inicial de respondentes, mas a decisão dos autores foi a de mantê-lo em seu formato original. Com a versão final do questionário concluída, partiu-se para o trabalho de campo (coleta de dados). O plano abrangeu duas estratégias de coleta: por meio de entrevistas pessoais e mediante a auto-aplicação do questionário, a uma amostra composta por 200 hóspedes da terceira idade, hospedados em hotéis de três capitais da região nordeste do país, durante os meses de abril e maio de 1998. 
De uma forma sistemática, o procedimento adotado para a escolha da amostra foi o seguinte, de acordo com as recomendações de Malhotra (1993).

- Definição da população. O primeiro passo foi definir a população da pesquisa, que foi assim constituída: homens ou mulheres, brasileiros, com 55 anos ou mais de idade, hospedados em hotéis de 2 a 5 estrelas, localizados em três capitais nordestinas, durante os meses de abril e maio de 1998.

- Moldura da amostra. Para uma delimitação da amostra, utilizou-se a ficha de identificação dos hóspedes, no caso da auto-aplicação dos questionários, e o julgamento do entrevistador para a escolha dos respondentes, no caso da aplicação através de entrevista pessoal.

- Técnica de amostragem. A técnica para a escolha da amostra foi de natureza não probabilística. Embora as considerações estatísticas sejam desfavoráveis para este procedimento e não recomendáveis quando se trata de um estudo descritivo, existiu a favorabilidade operacional, que foi o fator decisivo para a escolha desta técnica.

- Tamanho da amostra. Para a definição do tamanho da amostra, os autores basearam-se na média das amostras utilizadas em estudos similares, ficando em torno de 150 a 200 elementos (Holbrook, 1978; Westbrook, 1987; Oliver e Swan, 1989; Spreng, Mackenzie e Olshavsky, 1996).

No final do processo de coleta de dados estava-se com 155 questionários. Uma análise preliminar deles levou à exclusão de 12 questionários por motivos como estes: vários blocos incompletos e em 3 questionários o padrão de resposta pareceu inconsistente. O número de questionários passíveis de análises ficou em 143. Dos 143 questionários selecionados para a elaboração do banco dos dados, aproximadamente $25 \%$ apresentou o bloco referente ao construto atribuição incompleto ou totalmente não respondido. Por este motivo, o referido construto foi excluído da análise SEM.

O diagnóstico encontrado para este fato reside no não entendimento do bloco do questionário, que mensurou a atribuição pela totalidade dos respondentes. Apenas os questionários aplicados em entrevistas pessoais conseguiram índices de respostas satisfatórias. Os questionários respondidos mediante autopreenchimento ficaram comprometidos pela grande freqüência de não respostas. Assim, a decisão dos autores foi a de excluir este construto das análises, mesmo considerando a possibilidade de perda de informações. A insistência em mensurar a atribuição, mesmo após terem-se detectado problemas na fase do pré-teste, é explicada pela intenção de avaliarem-se simultaneamente todos os determinantes da satisfação propostos na literatura, embora o efeito da atribuição pareça 
indireto na resposta de satisfação. Além disso, nem sempre os consumidores processam esta dimensão no consumo de produtos e/ou serviços. O próximo tópico apresenta o resultado das análises realizadas.

\section{Análise dos Dados com a Modelagem de Equaçōes Estruturais (SEM)}

O diagrama apresentado na Figura 2 ilustra as relações propostas, bem como a magnitude dos efeitos diretos e indiretos entre as dimensões consideradas para esta etapa da análise dos dados. O diagrama foi desenhado com base no esquema teórico da satisfação do consumidor proposto por Oliver (1997), apresentado anteriormente.

\section{Figura 2: Diagrama da Análise de Equações Estruturais Padronizado}

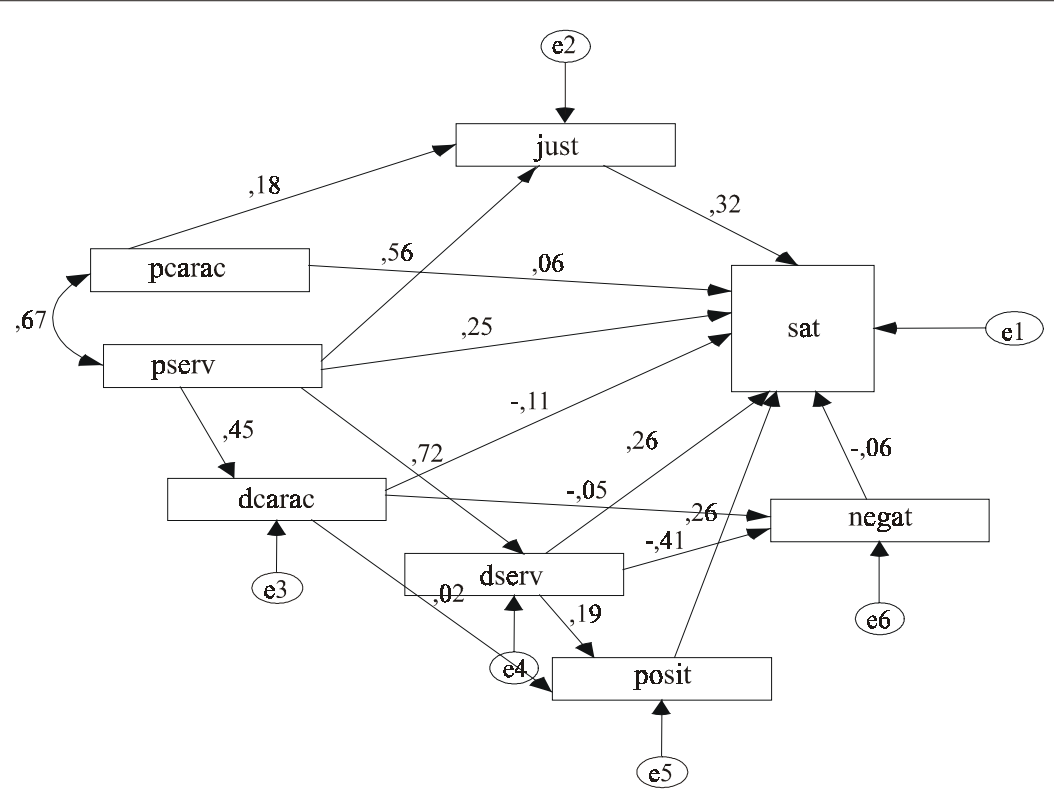

Fonte: análise de equações estruturais com o uso do Amos Graphics.

Todos os coeficientes dos caminhos (pesos beta) são padronizados.

Qui-quadrado $=244.087(\mathrm{DF}=12 ; \mathrm{p}=0,000)$.

Utilizando as especificações de Arbuckle (1997), Hair Jr. et al. (1998), Loehlin (1998) e Maruyama (1998), as variáveis presentes na análise de caminhos são classificadas da forma apresentada no Quadro 1, a seguir. 


\section{Quadro 1: Variáveis do Diagrama da Análise de Equações Estruturais}

\begin{tabular}{lll}
\hline Just (justiça - dimensão da eqüidade) & observada & endógena \\
Dserv (desconfirmação serviços) & observada & enógena \\
Dcarac (desconfirmação características) & observada & enóga \\
Negat (emoção negativa) & observada & enógena \\
Posit (emoção positiva) & observada & endógena \\
Sat (satisfação) & observada & enógena \\
Pcarac (performance características) & observada & exógena \\
Pserv (performance serviços) & observada & exógena \\
e1 (erro 1) & não observada & exógena \\
e2 (erro 2) & não observada & exógena \\
e3 (erro 3) & não observada & exógena \\
e4 (erro 4) & não observada & exógena \\
e5 (erro 5) & não observada & exógena \\
e6 (erro 6) & não observada & exógena \\
\hline
\end{tabular}

Fonte: resultado da análise de equações estruturais com o uso do Amos.

De forma sintética, o número de variáveis que compõem o esquema especificado na Figura 2 é igual a 14, sendo que 8 são observadas, 6 são não observadas, 8 são exógenas e 6 são endógenas. $\mathrm{O}$ modelo em análise é considerado recursivo. Os resultados encontrados com a utilização do Amos Graphics, indicaram um Qui-quadrado igual a $244.087(\mathrm{DF}=12, \mathrm{p}=0,000)$. O índice de adequação de ajustamento ajustado (AGFI) ficou em 0.258 , refletindo que o modelo carece de ajustamentos. O número de parâmetros estimados ficou em 24. Considerando o recomendado por Hair Jr. et al. (1998), onde estes autores citam que se deve ter de 5 a 10 casos por parâmetro estimado, a amostra utilizada de 143 elementos estaria considerada dentro do recomendado. Quando se multiplica 24 por 5, obtêm-se 120, indicando que o tamanho ideal da amostra deveria ser de no mínimo 120 casos, e aqui conseguiram-se 143. Estes mesmos autores afirmam que um número entre 100 e 150 elementos é suficiente, quando se utiliza o critério de máxima verossemelhança no modelo de regressão.

Para o cálculo dos coeficientes estimados das relações causais, utilizou-se a máxima verossemelhança, o que é comum neste tipo de análise (Patterson, 1993), e utilizada em modelos de regressão. Os dados apresentados na Figura 2 são os coeficientes de regressão padronizados (betas), também denominados coeficientes path.

Surpreendentemente, o efeito direto com maior impacto na resposta de satisfação foi o da variável justiça (dimensão da eqüidade, presente no esquema teóri- 
co analisado), por apresentar um coeficiente de regressão padronizado igual a 0,32 . Em segundo lugar surgiu o efeito das variáveis dserv (dimensão da desconfirmação, referente aos serviços do hotel) e posit (dimensão das emoções positivas), com um beta padronizado correspondendo a 0,26 . A dimensão pserv (dimensão da performance referente aos serviços do hotel) teve o coeficiente path correspondendo a 0,25 .

Oliver e Swan (1989) citam que os consumidores em uma transação se sentirão eqüitativamente tratados e assim satisfeitos, se a razão entrada/saída é de alguma forma justa. Ou seja, os indivíduos esperam receber o que é certo ou o que merecem pelo que estão pagando. De outro lado, autores como Moschis (1992) lembram que há uma crença de que os consumidores da terceira idade sejam benevolentes, preferindo ser recompensados abaixo do que seria considerado justo e, assim, o efeito de causa da eqüidade na satisfação não existira. Os resultados encontrados aqui revelam que isto pode não ser verdade, pois o efeito direto da dimensão justiça na satisfação corresponde a um beta de 0,31 (o maior de todos).

Complementando o parágrafo anterior, o resultado do trabalho empírico de Oliver e Swan (1989) revelou que os efeitos da eqüidade e da preferência não são claros na predição da satisfação. Quando mensurado juntamente com a desconfirmação, esta capta melhor a explicação da satisfação do que a eqüidade. A memória dos indivíduos limita a quantidade de informações que serão processadas em um dado momento e, por isso, nem todos os consumidores utilizam os conceitos de eqüidade. Além disso, indivíduos benevolentes, que preferem ser recompensados abaixo do que seria justo, parecem ser menos sensíveis às questões relativas à eqüidade. Assim, o efeito deste construto seria mínimo ou inexistente. Os resultados do presente estudo indicam caminhos diferentes, uma vez que a dimensão da justiça contribuiu de forma relevante na explicação da satisfação. $\mathrm{O}$ impacto direto da justiça sobre a resposta de satisfação pode também indicar que os consumidores idosos são experientes e buscam desenvolver estratégias de compra efetivas (Moschis, 1992), com base nos anos de experiência que possuem. Vale ressaltar que $74 \%$ dos respondentes nesta pesquisa viajam e hospedam-se em hotéis todos os anos, o que pode contribuir para o conhecimento dos atributos do serviço de hotelaria, reforçando a consciência do valor de uma troca justa.

O diagrama da Figura 2 representa um modelo recursivo de análise de caminhos, considerando que o fluxo das setas é sempre da esquerda para a direita, sem feedback. Para identificar o modelo e os possíveis erros de mensuração, as variáveis e1, e2, e3, e4, e5 e e6 foram acrescentadas. Estes erros são classificados como variáveis exógenas não observadas. $\mathrm{O}$ parâmetro fixado para estas 
variáveis foi igual a 1 (Arbuckle, 1997; Loehlin, 1998). A fixação de parâmetros faz-se necessária na identificação do modelo, correspondendo ao coeficiente de regressão.

\section{Figura 3: Diagrama da Análise de Equações Estruturais não Padronizado}

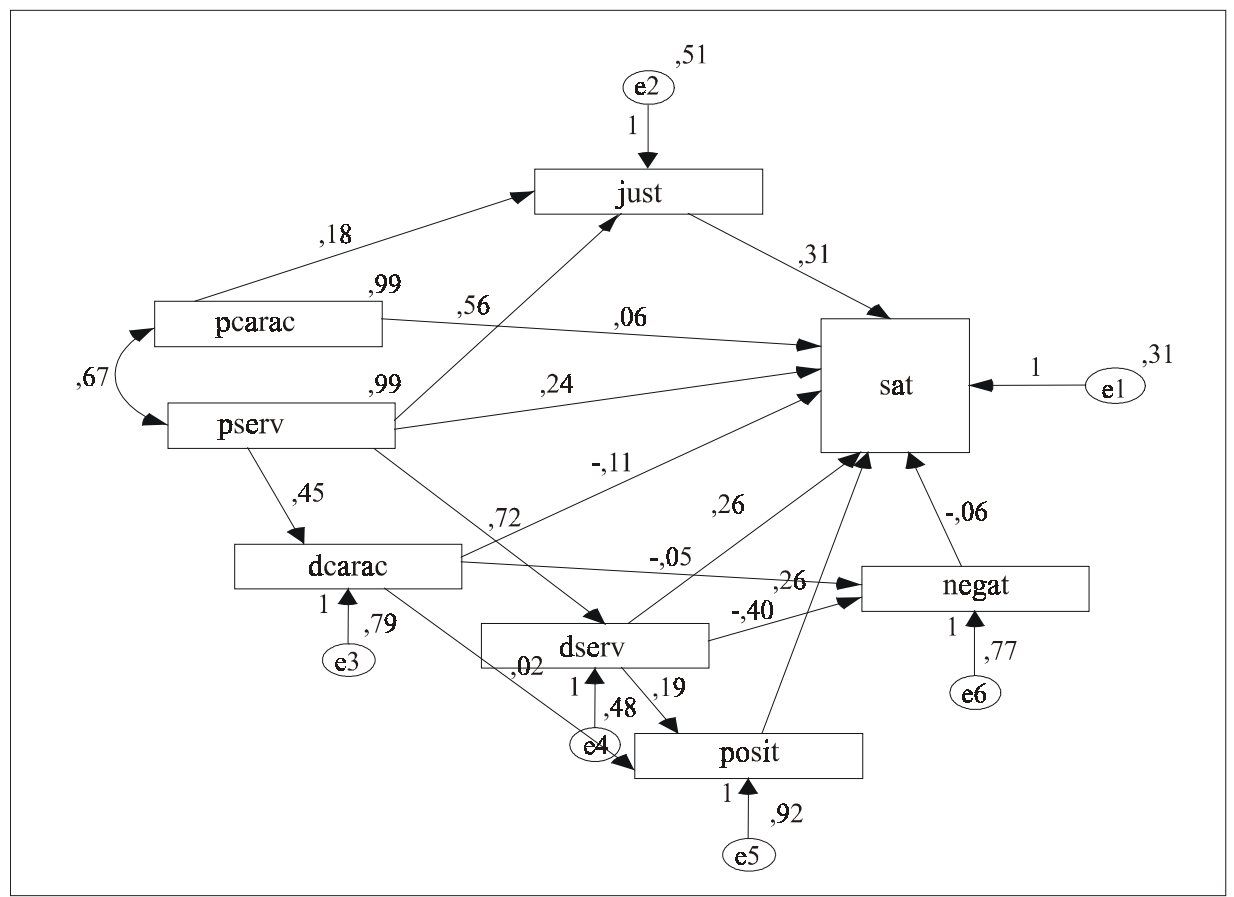

Fonte: análise de equações estruturais com o uso do Amos Graphics.

Todas as estimativas apresentadas são não padronizadas.

Qui-quadrado $=244.087(\mathrm{DF}=12 ; \mathrm{p}=0,000)$.

As estimativas apresentadas na Figura 3 são não padronizadas. Observa-se que o erro e1 explica $31 \%$ da variância do construto satisfação. Ou seja, os determinantes da satisfação incluídos na análise de equações estruturais deixam de explicar esta quantidade da variância da satisfação. Ressalta-se que para termos de identificação dos determinantes mais importantes, o que interessa são os resultados mostrados na Figura 2, onde estão presentes os coeficientes de regressão padronizados.

Embora a justiça se tenha apresentado como a variável com maior efeito direto na análise de equações estruturais, calculando-se o efeito total (influência direta e indireta) das variáveis no construto satisfação, com o uso do Amos, a 
performance dos serviços ofertados pelos hotéis (pserv) foi a variável que mais contribuiu para causar a satisfação (é parte da dimensão performance). O efeito total desta variável na satisfação foi de 0,61. Oliver e DeSarbo (1988) encontraram que a performance dos serviços ou produtos é um dos antecedentes, que apresenta maiores contribuições na determinação desta resposta. Possivelmente os consumidores pesquisados têm consciência da qualidade do serviço e avaliaram a performance adequada dos serviços dos hotéis (por exemplo, atendimento e serviço de copa) como sendo essencial para a satisfação. Relacionado a isto, Price, Arnould e Tierney (1995) citam que a performance oferecida ao cliente afeta claramente os sentimentos positivos vivenciados na experiência de consumo. O efeito total da performance dos serviços na satisfação é de 0,61 , como já mencionado, e das características do serviço é de 0,11 . O efeito direto destas variáveis na satisfação é respectivamente 0,25 e 0,06.

O efeito total da dimensão desconfirmação dos serviços foi o segundo maior $(0,33)$. A desconfirmação das características dos serviços teve uma influência negativa na satisfação $(-0,10)$. Churchill Jr. e Surprenant (1982) afirmam que, aparentemente, é a magnitude do efeito da desconfirmação que gera a satisfação. A desconfirmação positiva seria essencial para a existência da satisfação (Oliver, 1997). O forte impacto da performance na satisfação deve-se, primariamente, por este construto ser o que apresenta maior número de influência indireta na satisfação. Em outras palavras, a satisfação dos consumidores pesquisados com os serviços de hotelaria é determinada em grande parte pela percepção do desempenho dos serviços ofertados: atributos referentes à agradabilidade, limpeza, atendimento, copa, conforto e infra-estrutura. Os atributos relativos ao fator características, como localização, preço e segurança, também contribuem para a resposta de satisfação, embora em menor proporção.

Patterson (1993) afirma que a satisfação do consumidor é um fenômeno póscompra; assim, reflete o quanto o consumidor gosta ou não gosta de um produto, após o uso. O desempenho é fator crucial nesta avaliação. Isto também pode explicar o efeito total de a performance ter-se sobressaído ao das demais variáveis.

A terceira variável com maior impacto na satisfação é a justiça, dimensão da eqüidade, com um coeficiente total de 0,31 , já comentado. A justiça foi a única dimensão a entrar no diagrama para a análise de caminhos, apresentando uma contribuição significativa na determinação da satisfação.

As emoções positivas apresentaram um efeito total de 0,26 e as negativas contribuíram negativamente $(-0,06)$. $\mathrm{O}$ efeito da desconfirmação dos serviços nas emoções positivas foi de 0,19. Já nas emoções negativas esta mesma variável teve um coeficiente de regressão igual a $-0,41$. Isto pode indicar que os consumidores maduros são criteriosos na avaliação dos serviços; um desempenho abai- 
xo do esperado desperta sentimentos negativos, que podem ter impacto negativo na resposta de satisfação.

As emoções positivas são as que mais contribuem na resposta de satisfação; o efeito direto da dimensão emoção positiva nesta variável corresponde a um beta de 0 ,26. Já a dimensão das emoções negativas tem efeito negativo considerado baixo, tendo em vista que o coeficiente de regressão foi igual a $-0,06$, conforme explicitado no parágrafo anterior. A Tabela 1 apresenta os resultados totais das variáveis presentes no diagrama da análise de equações estruturais, obtidas com o comando total effects do Amos. Este cálculo pode também ser feito manualmente, seguindo as recomendações de Maruyama (1998) e Loehlin (1998), entre outros autores.

Tabela 1: Efeito Total das Variáveis na Satisfação

\begin{tabular}{|l|l|l|l|l|l|l|l|}
\hline Variáveis & Pserv & Pcarac & Dcarac & Dserv & Pos it & Negat & Just \\
\hline Dcarac & 0,453 & 0,000 & 0,000 & 0,000 & 0,000 & 0,000 & 0,000 \\
\hline Dserv & 0,720 & 0,000 & 0,000 & 0,000 & 0,000 & 0,000 & 0,000 \\
\hline Posit & 0,148 & 0,000 & 0,021 & 0,192 & 0,000 & 0,000 & 0,000 \\
\hline Negat & $-0,311$ & 0,000 & $-0,050$ & $-0,401$ & 0,000 & 0,000 & 0,000 \\
\hline Just & 0,562 & 0,179 & 0,000 & 0,000 & 0,000 & 0,000 & 0,000 \\
\hline Sat & $\mathbf{0 , 6 1 4}$ & $\mathbf{0 , 1 1 8}$ & $\mathbf{- 0 , 0 9 7}$ & $\mathbf{0 , 3 3 1}$ & $\mathbf{0 , 2 5 5}$ & $\mathbf{- 0 , 0 6 0}$ & $\mathbf{0 , 3 1 2}$ \\
\hline
\end{tabular}

Fonte: análise de equações estruturais com o uso do Amos Graphics.

A última linha da tabela resume o efeito total das variáveis performance (características e serviços), desconfirmação (características e serviços), emoções (positivas e negativas) e eqüidade (justiça). Conforme mencionado anteriormente, a variável Pserv apresenta o maior impacto na satisfação $(0,61)$, seguida pela Dserv $(0,33)$, Just $(0,31)$ e Posit $(0,26)$.

\section{ConClusÃo}

Os determinantes da satisfação do consumidor considerados nesta pesquisa foram: expectativas, performance, desconfirmação, atribuição, eqüidade e emoções. Estes construtos foram avaliados simultaneamente junto ao segmento da 
terceira idade que consome serviços de hotelaria, resultando em um trabalho inédito na literatura do marketing e do comportamento do consumidor no Brasil.

Foi possível verificar que nem todas as dimensões (determinantes) contribuem de maneira significante na explicação da satisfação. Já após a realização do pré-teste do questionário, excluiu-se o construto expectativas, por imaginar-se que os consumidores da terceira idade podem apresentar problemas de memória, uma das prováveis conseqüências do processo de envelhecimento, dificultando relembrar as expectativas (Moschis, 1992). Problemas de compreensão por parte dos respondentes ocorreram na mensuração da atribuição; assim, esta dimensão não foi incluída nas análises, a exemplo das expectativas.

Os quatro determinantes da satisfação incluídos na etapa da análise de equações estruturais foram a performance e a desconfirmação, cada um constituído por dois fatores relacionados às características e aos serviços dos hotéis. A eqüidade foi representada pelo fator justiça e as emoções no consumo foram compostas pelos fatores emoções positivas e negativas.

Os determinantes da satisfação estão interrelacionados e supõe-se que todos contribuem de algum modo para a explicação da satisfação, direta ou indiretamente. Isto foi comprovado com o resultado das análises multivariadas, embora nem todos os fatores/dimensões contribuam igualmente para a resposta de satisfação da amostra utilizada.

A performance surgiu como a dimensão que melhor explicou a satisfação, com o fator relacionado aos serviços dos hotéis apresentando um coeficiente de regressão maior do que o formado pelas características dos hotéis. A segunda dimensão mais relevante para a determinação da satisfação dos consumidores da terceira idade foi a desconfirmação, considerando o efeito total deste construto. Mais uma vez, o fator relacionado aos serviços dos hotéis se sobressaiu.

A terceira variável que mais influenciou a resposta de satisfação nesta pesquisa foi o fator justiça, da dimensão da eqüidade. Na verdade, a maior influência direta na satisfação foi desta variável (coeficiente de regressão padronizado igual a 0,32 ). Aparentemente, os consumidores maduros pesquisados valorizam uma relação de troca justa.

As emoções no consumo representadas pelos fatores emoções positivas (posit), e negativas (negat), estão em quarto lugar na determinação da resposta de satisfação. $O$ efeito das emoções positivas é significativo (coeficiente de regressão padronizado igual a 0,26 ) e positivamente relacionado à satisfação. Já as emoções negativas apresentaram uma relação negativa praticamente insignificante (beta igual a $-0,06$ ). 
Conclui-se que os determinantes que mais contribuem para a explicação da resposta de satisfação no segmento da terceira idade no Brasil, no que concerne ao consumo de serviços de hotelaria são: performance, desconfirmação, justiça e emoções.

Destaca-se o fato de que o estudo apresenta limitações, entre as quais cumpre incluir: o índice AGFI não foi considerado adequado; problemas no bloco do questionário que mensurava a atribuição, fizeram com que este construto fosse excluído das análises, conforme se mencionou anteriormente. As expectativas também não foram mensuradas.

\section{Sugestões para Futuras Pesquisas}

Especificamente, os autores propõem três desafios para futuros estudos.

- Reduzir o tamanho do instrumento de coleta de dados, para que mensure todos os determinantes da satisfação, sem que haja perda de informações em um único estudo.

- Desenvolver uma escala para mensurar a dimensão da atribuição, de modo que possibilite melhor compreensão para o segmento da terceira idade, com base em uma revisão teórica do tema.

- Reaplicar o estudo em outras situações de compra, na tentativa de avaliar realmente todos os determinantes da satisfação em um único estudo, mesmo que de forma longitudinal, mensurando as dimensões isoladamente em uma série de estudos.

\section{Nota}

${ }^{1}$ A versão completa do questionário com a operacionalização das dimensões pode ser solicitada aos autores da pesquisa. A dimensão expectativa foi excluída devido à problemas de viés na coleta dos dados e às controvérsias deste paradigma relacionadas ao segmento de consumidores pesquisado (e.g., Moschis, 1992). 
ReferênCias Bibliográficas

ARBUCKLE, J. L.

AMOS users' guide : version

3.6. Chicago, IL : SPSS, 1997.

BENTLER, P. M.

EQS : structural equation manual. Los Angeles, CA : BMDP Statistical Software, 1989.

BITNER, M. J.;

NYQUIST, J. D.;

BOOMS, B. H.

The critical incident technique for analyzing the service encounter. In: Services marketing in a change environment. Chicago, IL : American Marketing Association, 1985. proceedings series. p. 48-51.

CARDOZO, R. N.

An experimental study of customer effort, expectation, and satisfaction. Journal of Marketing Research, v. II, p. 244-249, Aug. 1965.

CHURCHILL JR., G. A.;

SURPRENANT, C.

An investigation into the determinants of customer satisfaction. Journal of Marketing
Research, v. XIX, p. 491-504, Nov. 1982.

CZEPIEL, J. A.;

ROSENBERG, L. J.;

SURPRENANT, C.

The development of thought, theory, and research in consumer satisfaction. In: LAMB JR., C. W.; DUNNE, P. M. Theoretical developments in marketing. Chicago, IL : American Marketing Association, 1980. p. 216-219.

EWALD, R.

Satisfação do consumidor : integração entre teoria e prática. São Paulo, 1993. Dissertação (Mestrado em Administração) Escola de Administração de Empresas de São Paulo, Fundação Getúlio Vargas.

FARIAS, S. A. et al.

Emoções e sentimentos na resposta de satisfação dos consumidores : o caso de um programa de MBA. In: XXI ENCONTRO ANUAL DA ANPAD (1997: Angra dos Reis). Anais Eletrônicos... Rio de Janeiro : ANPAD, 1997. 
FARIAS, S. A.;

SANTOS, R. DA C.

Atributos de satisfação nos serviços de hotelaria : uma perspectiva no segmento da terceira idade. In: XXII ENCONTRO ANUAL DA ANPAD (1998 : Foz do Iguaçu). Anais Eletrônicos... Foz do Iguaçu : ANPAD, 1998.

FLANAGAN, J. C.

The critical incident technique. Psychological Bulletin, v. 51, p. 327-357, July 1954.

FOURNIER, S.;

MICK, D. G.

Rediscovering satisfaction. Journal of Marketing, v. 63, October 1999.

GRANDIS ROJO, F. J.

O comportamento do consumidor nos supermercados. Revista de Administração de Empresas, v. 38, n. 3, 1998.

HAIR JR., J. F. et al.

Multivariate data analysis with readings. 4. ed. Englewood Cliffs, NJ : Prentice-Hall, 1995.

Multivariate data analysis. 5. ed. Englewood Cliffs, NJ : Prentice-Hall, 1998.
HOLBROOK, M. B.

Beyond attitude structure : toward the informational determinants of attitude. Journal of Marketing Research, v. XV, p. 545-556, Nov. 1978.

IZARD, C. E.

Human emotions. New York : Plenum Press, 1977.

JÖRESKOG, K. G.;

SÖRBOM, D.

LISREL 8 : structural equation modeling with simplis command language. Mooresville, IN : Scientific Software, 1993.

KLEM, L.

Path analysis. In: GRIMM, L. G.; YARNOLD, P. R. Reading and understanding multivariate statistics. Washington, DC : American Psychological Association, 1995.

LOEHLIN, J. C.

Latent variables models : an introduction to factor, path and structural analysis. 3. ed. Mahwah, NJ : Lawrence Erlbaum, 1998.

MALHOTRA, N. K.

Marketing research : an applied orientation. Englewood Cliffs, NJ : Prentice-Hall, 1993. 
MARUYAMA, G. M.

Basics of structural equation modeling. London : Sage Publications, 1998.

MOSCHIS, G. P.

Marketing to older consumers : a handbook of information for strategy development. Westport, CT : Quorum Books, 1992.

\section{OLIVER, R. L.}

Theoretical bases of consumer satisfaction research : review, critique, and future directions. In: LAMB JR., C. W.; DUNNE, P. $M$. Theoretical developments in marketing. Chicago, IL : American Marketing Association, 1980. p. 206-210.

Satisfaction : a behavioral perspective on the consumer. New York: McGraw-Hill, 1997.

OLIVER, R. L.;

DESARBO, W. S.

Response determinants in satisfaction judgments. Journal of Consumer Research, v. 14, p. 495-507, Mar. 1988.

OLIVER, R. L.;

SWAN, J. E.

Equity disconfirmation perceptions as influences on merchant and product satisfaction. The Journal of Consumer Research, v. 16, p. 372-383, Dec. 1989.
PARASURAMAN, A.;

ZEITHAML, V. A.;

BERRY, L. L.

A conceptual model of service quality and its implication for future research. Journal of Marketing, v. 49, p. 41-50, Autumn 1985.

PATTERSON, P. G.

Expectations and product performance as determinants of satisfaction for a high-involvement purchase. Psychology \& Marketing, v. 10, n. 5 , p. 449-465, Sept./Oct. 1993.

PRADO, P. H. M.;

FARIAS, S. A.

Avaliação da atribuição e da desconfirmação como antecedentes da resposta de satisfação dos consumidores com serviços bancários. In: XXII ENCONTRO ANUAL DA ANPAD (1998 : Foz do Iguaçu). Anais Eletrônicos... Foz do Iguaçu : ANPAD, 1998.

PRADO, P. H. M.;

MARCHETTI, R.

Excelência em supermercados : a dimensão da satisfação do consumidor. Revista de Adminstração, v. 32, n. 2, abr./jun. 1997. 
PRICE, L. L.;

ARNOULD, E. J.;

TIERNEY, P.

Going extremes : managing service encounters and assessing provider performance. Journal of Marketing, v. 59, p. 83-97, Apr. 1995.

ROSSI, C. A. V.;

SLONGO, L. A.

Pesquisa de satisfação de clientes : o estado-da-arte e proposição de um método brasileiro. In: XXI ENCONTRO ANUAL DA ANPAD (1997 : Angra dos Reis). Anais Eletrônicos... Rio de Janeiro : ANPAD, 1997.

SPRENG, R. A.;

MACKENZIE, S. B.;

OLSHAVSKY, R. W.

A reexamination of the determinants of consumer satisfaction. Journal of Marketing, v. 60, p. 15-32, July 1996.

SPSS.

SPSS base 8.0 user's guide. Chicago : SPSS Inc., 1998.
URDAN, A. T.;

RODRIGUES, A. R.

O modelo de índice de satisfação do cliente norte-americano: um exame inicial no Brasil com equações estruturais. In: XXII ENCONTRO ANUAL DA ANPAD (1998 : Foz do Iguaçu). Anais Eletrônicos... Foz do Iguaçu : ANPAD, 1998.

WESTBROOK, R. A.

Product/consumption-based affective responses and postpurchase processes. Journal of Marketing Research, v. XXIV, p. 258-270, Aug. 1987.

WESTBROOK, R. A.;

OLIVER, R. L.

The dimensionality of consumption emotion patterns and consumer satisfaction. Journal of Consumer Research, v. 18, p. 84-91, June 1991.

YI, Y.

A critical review of consumer satisfaction. In: ZEITHAML, V. A. (Ed.). Review of marketing 1990. Chicago, IL : AMA, 1991. p. 68-123. 